\title{
EL INFINITIVO GRIEGO: UN INTENTO DE RECONSTRUCCIÓN
}

EnRIQue García Domingo

1. El inf. gr. presenta una gran variedad formal1. Trataremos, a continuación, de explicar sus formaciones más características.

2. Aunque el método comparativo pone de manifiesto la indudable antigüedad de algunas formaciones de inf., ninguna de ellas cubre todo el ámbito ide. Los variados testimonios de los dialectos ide. hacen, pues, imposible restituir una forma única para la lengua común 2 . Tal vez, por ello, sea más adecuado, para comprender el inf. ide., recurrir a la teoría de las ondas (Wellentheorie): a partir de una lengua ide., una característica o marca de inf. se habría expandido a otra $u$ otras originando una isoglosa ${ }^{3}$.

El inf. gr. - $\varepsilon v$ no tiene correspondencia exacta en los demás dialectos ide., pero su adscripción a la etapa ide. se deduce no sólo porque se encuentra

1 E. SCHWYZER, Griechische Grammatik, I, München 19684, 804-810; P. Chantraine, Morphologie historique du grec, Paris 19612, 273-280; H. Rix, Historische Grammatik des Griechischen. Darmstad 1976, 237-239. Un resumen de las opiniones más importantes sobre el infinitivo griego hasta las fechas indicadas puede verse en L. Macía, «Cuatro cuestiones de morfología griega», Actualización cientifica en Filología Griega, Universidad Complutense, Madrid 1984, 313-319; A. BERNABÉ, «La morfología griega en los últimos años», E. Clas., XXXI, 95, 1989, 59, 60.

2 F. VILLAR, Los indoeuropeos y los origenes de Europa, Madrid 1991, 256.

3 Sobre el parentesco lingüístico vid. E. VILLAR, op. cit., 469-484. 
como segmento final en el inf. véd. -san(i), - $\operatorname{man}(e)$ y gr. $-\mu \varepsilon v$, sino también porque es la base de otras formaciones nominales posteriores con vocal temática, *-ê/ŏ́n-o: ai. adj. verbal vahanas, part. pres. med. atem. bhindãnas; gót. inf. pres. bairan, part. pret. pas. fulgins, bundans; aesl. part. pret. pas. nesenü.

En cambio, el gr. comparte con el i.-ir. la isoglosa del inf. *-men: gr. $\gamma \sim \tilde{\omega} \mu \varepsilon v$, véd. dāmane (<*-men-ei, dat. sg.). Este inf. es, asimismo, el punto de partida de otras formaciones nominales temáticas, *-mĕn-o-: ai. part. pres. med. tem. bharamānas, gr. $\varphi \varepsilon \rho 6 \mu \varepsilon v o \zeta$, los restos lat. alumnus, femina (<*-menā) y quizá luv. part. pas. kes-ama-4.

3. Estos dos alomorfos eran las formas más simples y más antiguas de que disponia, para expresar el inf., este grupo de indoeuropeos que al cabo de varias generaciones iba a terminar hablando griego: a) inf. *-en tras temas verbales en consonante, sonante consonante y vocales $\overline{\mathrm{i}}, \overline{\mathrm{u}}$; $\mathrm{b}$ ) inf. *-men tras temas verbales en vocal (inclusive $\breve{\mathrm{i}}, \breve{\mathrm{u}}$ ) o sonante vocálica.

La presencia de *-en tras $i$, $u$ era posible porque estas vocales desarrollaban ante otra vocal una breve consonante de transición iye, uwe (glidesound), tal y como demuestran algunos dialectos ide. en época histórica5: panf. uع$v \propto l$, Eret. $\delta v F \varepsilon$, ai. inj. aor. śruvam, pres. med. iye «voy», etc. Si las vocales eran $\bar{i}$ y $\bar{u}$, se desdoblaban en iy y uw6: ai. aor. abhuvam (2.a sg. a-bhïs), gen. dhiyas (nom. dhis), pero la cantidad larga podía ser luego restituida por influencia de los casos en que $\overline{\mathbf{i}}$ y $\overline{\mathrm{u}}$ aparecían ante consonante: ai. aor. $a b h^{-}$ uvam, gr. gen. Kĩos (nom. Ki $)^{7}$.

He aquí, pues, la situación primitiva ${ }^{8}$ :

4 Acerca de las mencionadas formaciones nominales vid. K. BRUGManN-B. DeLBRUCK, Grundriss der vergleichenden Grammatik der indogermanischen Sprachen, Strassburg 1897-1916, II, 1, 230-232, 241, 266-268, 638-640, 651; II, 2, 167, 178; II, 3, 899; A. A. MACDONELL, Vedic grammar, Strassburg 1910, 313, 325, 326, 339, 340, 362, 364, 410. O. SZEMERENYI, Introducción a la lingülstica comparativa, Madrid 1978, 404, 405, 408-411.

5 M. LEJEUNE, Phonétique historique du mycénien et du grec ancien, Paris 1972, 163, 164 , 245; A. THUMB-R. HaUSCHILD, Handbuch des Sanskrit, 1.1 Heidelberg 1958, 227, 228.

6 En estos casos las vocales largas siempre se transforman en breves, porque los órganos articulatorios tienen que desplazarse rápidamente para articular la breve consonante de transición que sigue.

7 A. ThUMB-R. HAUSCHILD, op. cit., 1.2 (1959), 297; E. SCHWYZER, op. cit., 570, 571.

8 Respecto a la etimologfa de los verbos utilizados consúltese J. POKORNY, Indogermanisches etymologisches Wörterbuch, I, Bem 1959; H. FRISK, Griechisches etymologisches Wörterbuch, Heidelberg I 1960, II, 1970, III, 1972; P. CHANTRAINE, Dictionnaire étymologique de la langue grecque, París 1968-1980.

Para los inf. dialectales, además de las gramáticas citadas, vid. A. THUMB-E. KIECKERS-A. SCHERER, Handbuch der griechischen Dialekte, I, II, Heidelberg 19322-19592. 

a) inf. *-en
b) inf. *-men
* bher-en (arc. $\varphi \hat{\varepsilon} \rho \varepsilon v$ )

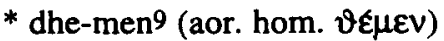

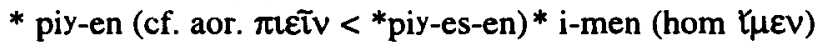

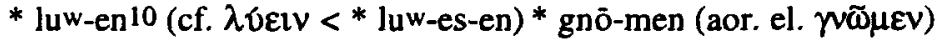
$*$ iy-en (arc. $\dot{\varepsilon} \xi \mathfrak{l} \varepsilon v)$

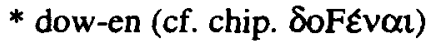

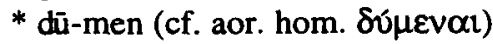
* weid-en (cf. pf. i $\delta \varepsilon ́ v \alpha l$ )

4. Cuando el mencionado grupo, antepasado de los griegos, establecio, dentro del sistema verbal, una oposición entre formaciones temáticas y atemáticas, reorganizó estos infinitivos de otra manera, colocando unos junto a las formaciones temáticas (la mayoría de los inf. en *-en) y otros al lado de las atemáticas (todos los en *-men y algunos en *-en). Esta redistribución del inf. gr. es de capital importancia porque ha provocado toda la evolución posterior del mismo.

La situación es ahora la siguiente:
a) Formaciones temáticas
1) inf. *-en
* bher-en (cf. $\varphi \in p \omega)$
* piy-en (cf. aor. Ł̇tıov)
* luw-en (cf. $\lambda v \omega$ )

\author{
b) Formaciones atemáticas \\ 2) inf. *-men \\ * dhe-men (cf. pres. $\tau\{\vartheta \eta \mu \mathrm{l}$ ) \\ * i-men (cf. pres. Ei $\tilde{\mu} \mu$ ) \\ * gnō-men (cf. aor. Eүvwv) \\ * dū-men (cf. aor. $\varepsilon \delta \bar{v}$ ) \\ * gwe-gwm-men (cf. pf. $\beta \varepsilon \beta \alpha \sigma \sigma l$ ) \\ 3) inf. *-en \\ * iy-en (cf. pres. Ei $\mu \mathrm{l}$ ) \\ * dow-en (cf. pres. $\delta \mathfrak{t} \delta \omega \mu \mathrm{l})$ \\ * weid-en (cf. pf. oĩ $\delta \alpha$ )
}

$9 \mathrm{Si}$, en la reconstrucción del ide., partimos de la teorfa de las ondas, podemos suponer que ai. hitás, gr. ७દws, lat. factus no coinciden exactamente y presentan tres vocales originarias distintas. En cambio, si tenemos «in mente» la imagen del árbol genealógico, las tres vocales derivarian de un fonema único, es decir, de una schwa o de la laringal $\mathrm{H}_{\mathrm{i}}$. $\mathrm{Y}$, de acuerdo con la teorfa larin-

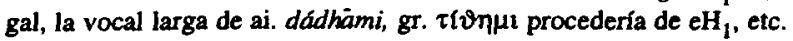

En cualquier caso, como el inf. es, según dijimos (2.), una creación reciente, no atribuible a la lengua común, habria que admitir que, tras la separación dialectal y una vez transformadas las laringales, los dialectos, todavía en contacto, habrían intercambiado los morfemas de infinitivo. Con lo que la teoría del árbol genealógico y la teoría de las ondas resultan totalmente compatibles y complementarias.

10 A juzgar por el lat. luō, solvó < "se-luo, $\lambda u ́ \omega$ es un presente radical temático. Sin embargo, en otros presentes gr. en - $v \omega$ es difícil determinar si son radicales tem. o comportan el sufijo de pres. *-ye-/*-yo-, cuya y solo se ha conservado en eolio: eol. $\varphi$ ut $\omega$ át. $\varphi v \omega$; eol. $\alpha \lambda v i \omega$, at. $\alpha \lambda \omega \omega$, etc. Vid. E. SCHWYZER, op. cit., I, 686; P. ChANTRAINE, Grammaire homérique, I, París 1958, 372374 y op. cit., 232. 
Los inf. de temas verbales en $-\breve{\breve{u}}$ y en $-\breve{\bar{l}}$ podían adaptarse fácilmente al nuevo sistema de una manera bien sencilla, escogiendo el alomorfo *-en para alinearse junto a las formaciones temáticas (lo que generalmente ocurrió), *-men junto a las atemáticas (como hicieron los demás temas en vocal).

Sin embargo, aquellos inf. de temas en consonante que, en lugar de permanecer en el sistema tem., debían integrarse en el atem., no tenían más opción que pasar a él con la característica *-en. Esta posibilidad, la de emplear *-en (además de *-men), también podía ser compartida por los inf. de temas verba-

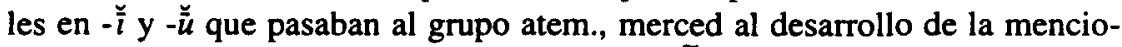
nada consonante de transición. Así el v. atem. Ei $\mu l$ presenta los dos alomorfos de inf., *i-men (hom. $\nmid \mu \varepsilon v)$ e *iy-en (arc. $\varepsilon \xi$ - $\{\varepsilon v)$.

El sistema de inf. descrito era inestable porque no permitía una distinción clara entre inf. temáticos y atemáticos. Como veremos, la lengua tratará de subsanar este inconveniente.

5.1. I.-ir. y lat. participan de la isoglosa del inf. en silbante11, cuya distribución primitiva debió de ser la siguiente: a) tras temas en vocal (inclusive $\bar{\imath}$, $\breve{\bar{u}}$ ), *-s, por ej., véd. jiṣe < *gwi-s-ei, stuse < *stu-s-ei (dat.), lat. fore < *bhu-s$\mathrm{i}$, dare <*da-s-i (loc.); b) tras temas en consonante y vocal $\breve{\mathrm{i}}, \overline{\mathrm{u}}, *$-es, por ej., véd. jīvase < *gwīw-es-ei, bhiyase < *bhiy-es-ei, lat. vivere < *gwiw-es-i.

Pues bien, la isoglosa del inf. *-(e)s-en que comparten ai. y gr. 12 es el resultado del intercambio de dos inf., ai. *-(e)s y gr. *-en.

En efecto, el inf. ai. en $*$-s, a causa de su extremada brevedad, fue hipercaracterizado por *-en, desinencia de inf. tomada del gr., resultando ${ }^{*}$-s-en: véd. bhüṣani < *bhū-sen-i, neșani < *nei-sen-i (loc.).

Por su parte el griego, con el fin de evitar la ambigüedad del inf. *-en, utilizado en tem. (*bher-en, arc. $\varphi \hat{\varepsilon} \rho \varepsilon v)$ y atem. (*iy-en, arc. $\varepsilon_{\xi}^{\xi}$ - $\varepsilon v$ ), tomó en préstamo del ai. la desinencia de inf. *-es, que hipercaracterizada por *-en (es decir, ${ }^{*}$-es-en 13) sirvió para distinguir los inf. tem. de los atem. ${ }^{*}$-en y ${ }^{*}$-men.

Sin embargo, el nuevo inf. tem. *-es-en tuvo que convivir también con el antiguo inf. tem. *-en. Como es sabido, una innovación lingüística se va abriendo paso poco a poco y tarda en consolidarse, porque en una comunidad

11 K. BRUGMANN-B. DelbrüCK, op. cit., II, 1, 142, 525, 531, 536, 537, 640; II, 2, 174, 184 ; II, 3, 899; A. A. MACDONELL, op. cit., 409; LEUMANN-HOFMANN-SZANTYR, Lateinische Grammatik, I, München 1977, 580, 581.

12 K. Brugmann-B. Delbrúck, op. cit., II, 1, 311; A. A. MaCdonell, op. cit., 412; E. SCHWYZER, op. cit., I, 809.

13 Tanto éste como el ai. *-s-en pertenecen, pues, a lo que podriamos llamar segunda oleada de inf. No se trata ya de inf. simples (*-en, *-men, *-es, *-s), sino hipercaracterizados, compuestos de dos morfemas de inf., lo cual supone un estrato o nivel linguístico diferente. 
siempre hay individuos conservadores que continúan aferrados a las formas más arcaicas.

5.2.1. El inf. gr. med. $-\varepsilon \sigma \vartheta \alpha \mathrm{l}<*$-es-dhai 14 es una creación singular que no tiene correspondencia en otras lenguas ide. Sin embargo, cualquiera que haya sido su origen, que pertenece al mismo estrato o nivel lingüístico que el act. *-es-en lo podemos deducir no sólo por su coincidencia con el primer elemento del mismo, sino también, como más adelante veremos (6.), por sus implicaciones en la evolución posterior del inf. gr.

Vamos a intentar explicarlo, aunque para ello tengamos que dar un pequeño rodeo.

5.2.2. Como es sabido, el inf. es un sustantivo verbal que expresa esencialmente la idea del verbo. A diferencia del inf. véd. que ha desarrollado al-

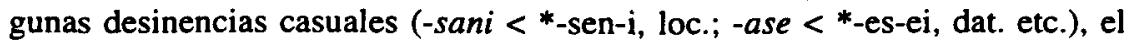
inf. gr. carece de ellas 15 . Por ello, el propio sufijo $\left(^{*}\right.$-en, *-men, *-es-en) fue considerado como desinencia, que coincidía en la forma, total o parcialmente, con uno de los alomorfos de 1.a pers. pl. act. del verbo (*-men).

La mencionada coincidencia formal no es un hecho circunstancial ni aislado, pues, según se ha observado ya, nombres y verbos presentan a veces sufijos y desinencias comunes: *-nt, *-(e)s y *(o)m16. En fin, a falta de desinencias casuales específicas, el inf. gr. pudo reforzar su carácter nominal gracias al apoyo del artículo.

De otro lado, los hablantes de este grupo pre-griego debían tener conciencia de que *(-o)-m-e-n $>-\mu \varepsilon v$, alomorfo de $1 .{ }^{a}$ pl. act., era una ampliación de $*(-0)-m>-v, 1$.a $\mathrm{sg}$. TS act.; por lo que *-en en la $1 .{ }^{*} \mathrm{pl}$. act. era un segmento aislable. De suerte que no sólo la 1.a pl. act. *-men y el inf. *-men coincidían, sino que además el inf. *-en (presente también en *-es-en) era aparentemente idéntico al segmento final utilizado para formar la 1. a $\mathrm{pl}$. act. a partir de la $1 . \mathrm{a}$ sg. TS act.17.

14 Las opiniones más importantes sobre este infinitivo están recogidas en L. Macía, art. cit., 316,317 . G. Cohen ( $($ Origin of greek - $\sigma v \alpha l », K Z, 95,1981,293-301$, no mencionado por $L$. Macía) vuelve a considerar la posibilidad de que $-\sigma \vartheta \alpha$ sea una desinencia primaria de 2 .a pl. med., reconvertida luego en desinencia de inf. med.

15 Sobre el inf. gr., además de las obras mencionadas, vid. Burguière, Histoire de l'infinitif en Grec, Paris 1965, 23-48.

16 F. R. ADRADOS, Lingülstica indoeuropea, Madrid 1975, 624, 742, 743, 908.

17 Las desinencias (y la vocal temática), antes de morfologizarse como tales, fueron simples alargamientos de la raíz o tema verbal. Así por ej.: *(-o)-m, 1.a sg. TS act.; *(-б̆)-m-e, *(-б̆)-m-es, *(-o)-m-e-n, *(-0)-m-0-s, 1.a pl. act.; *(-0)-m-e-dh-a, *(-o)-m-e-dh-a-i, *(-0)-m-e-dh-i, 1,a pl. med., etc. Cf. F. VILLAR, «Las desinencias verbales indoeuropeas de primera y segunda persona de plural», RSEL, 4, 1974, 391-409; F. R. ADRADOS, op. cit., 625. 


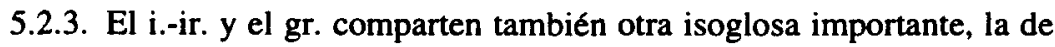
la voz media, cuyas desinencias, sin embargo, no siempre coinciden exactamente. A pesar de que puede haber en latín o celta alguna desinencia relacionable, se ha señalado ya que sería desacertado retrotraer el sistema representado por el i.-ir. y gr. a la lengua común 18 .

Resulta claro que las desinencias medias 2 a sg. *-so, *-soi, 3.a sg. *-to, *-toi y 3.a pl. *-nto, *-ntoi derivan de las TS act. correspondientes *-s, *-t y

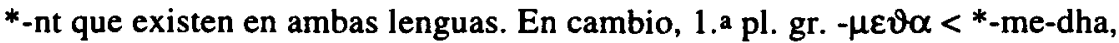
i.-ir. TP -mahe, -made <*-me-dhai, TS-mahi, -madi $<*$-me-dhi están construidas sobre la 1.a pl. TS act. *-me sólo conservada en i.-ir. -ma19. Que *-dha es un segmento aislable lo corrobora la 1.a pl. gr. $-\mu \varepsilon \sigma \vartheta \alpha$ formada sobre la act. $-\mu \varepsilon \varsigma^{20}$. Lo propio podemos decir de *-dhi, utilizado también como desinencias de imper. atem. 2.a sg. en ai. $(-h i)$ y gr. $(-\vartheta \imath)$, y de ${ }^{*}$-dhai, elemento final del inf. gr. med. $-\varepsilon \sigma-\vartheta \alpha$.

En efecto, cuando este grupo ide. antecesor de los griegos sintió necesidad de crear el inf. med., se fijó en el inf. act. *-es-en, cuyo segmento final *-en fue identificado con el de la 1.a pl. act. (5.2.2.). Según el modelo de inf. act. se formó *-es-dhai, cuyo segmento final *-dhai es el mismo que presenta la mencionada TP 1.a pl. med. del i.-ir. Es el tipo panhelénico tem. $\varphi \varepsilon \rho \varepsilon \sigma \vartheta \alpha$, del que deriva el atem. KẼ $\sigma \vartheta \alpha$.

5.3. La situación del inf. en este grupo antepasado de los griegos es ahora así:

a) Formaciones temáticas

b) Formaciones atemáticas

Activa

1) inf. *-en

*bher-en > arc. $\varphi € \rho \varepsilon v$, etc.

3) inf. *-es-en

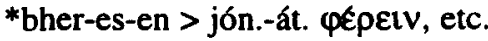

Media

5) inf. *-es-dhai

*bher-es-dhai $>\varphi \varepsilon \rho \varepsilon \sigma \vartheta \alpha$ l, etc.
2) inf. *-men

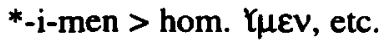

4) inf. *-en

$*$ iy-en $>$ arc. $\varepsilon \xi$ - $\varepsilon \varepsilon v$, etc.

6) inf. *-s-dhai

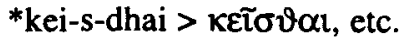

Las modificaciones posteriores se producirán en griego común, cuando este grupo no esté ya en contacto con ninguna rama de la comunidad ide.

18 F. VILLAR, op. cit., 250, 251.

19 Sobre estas desinencias vid. O. SZEMERENY1, op. cit., 301-309.

20 Acerca de estos alomorfos vid. E. SCHWYZER, op. cit., I, 662, 663, 670. 
6. Pero el sistema del inf. gr. seguía siendo inestable. Lejos de solucionar el problema, ${ }^{*}$-es-en se convirtió simplemente en un alomorfo del inf. tem., al seguir coexistiendo *-en en tem. y atem. La lengua recurrió entonces al recién creado inf. med. y extendió su secuencia final *-ai al inf. act. atem. (*-en-ai) para distinguirlo inequívocamente del tem. (*-en). Se trata de un proceso de diferenciación.

El panorama en la act. es ahora el siguiente:
a) Formaciones temáticas
b) Formaciones atemáticas
1) inf. *-en
2) inf. *-men
*bher-en > arc. $\varphi \in \rho \varepsilon v$, etc.
$*_{\text {i-men }}>$ hom. $\chi_{\mu \varepsilon v}$, etc.
3) inf. *-es-en
4) inf. *-en-ai

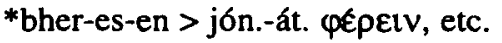
*iy-en-ai > jón.-át. léval, etc.

El paso posterior fue extender *-ai de *-en-ai al inf. *-men para caracterizar todo el grupo atem. frente al tem. que carecía de esta marca: inf. tem. act. *-en. *-es-en; atem. *-en-ai, *-men-ai (hom. Sú $\mu \varepsilon v \alpha l$, etc.). Es un proceso de igualación.

7. Ya en gr. común la $\varepsilon$ del inf. atem. - $\varepsilon v \propto r$ fue eliminada, por ser inne-

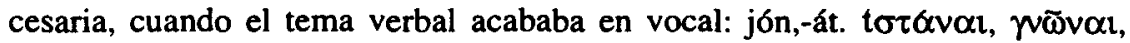
$\beta \varepsilon \beta\langle v \alpha$, etc.

$Y$, a la inversa, si - $\alpha \propto \mathfrak{l}$ podía aparecer tras temas en vocal, $-\mu \varepsilon v(\alpha \mathfrak{l})$ podría también hacerlo tras temas en consonante: tes. $̌ \mu \mu \varepsilon v$, hom. $₹ \delta \mu \varepsilon v$,

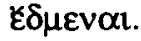

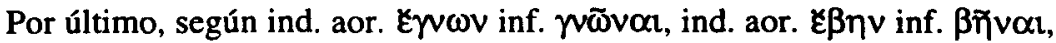
ind. aor. $̌ \delta u v$ inf. $\delta \tilde{v} v \alpha$, etc. (en donde - $\alpha$ l parecía ser una prolongación de 1.a sg. - $v$ ), se formó, a partir del ind. aor. 1.a sg. $-\sigma \alpha^{21}$, el inf. aor. - $\sigma \alpha$ que es panhelénico y el más reciente de todos: por ej. ind. $\varepsilon \delta € \imath \xi \alpha$, inf. $\delta \varepsilon \tau \xi \alpha$, etc. 22

8.1. De esta rica gama de infinitivos del gr. común, los dialectos escogieron un tipo u otro: jón.-át. tem. $-\varepsilon \imath \mathrm{v}$, atem. $-(\varepsilon) v \alpha$; arc. tem. $-\varepsilon v$, atem. $-(\varepsilon) v \alpha l$, tes. tem. $-\varepsilon \imath v$, lesb. $-\eta \nu\left({ }^{*}\right.$-es-en), tes. atem. $-\mu \varepsilon v$ lesb. $-\mu \varepsilon v \propto l$, etc.

8.2. Algunos inf. no son elecciones del mencionado acervo común de infinitivos griegos, sino innovaciones compartidas por algunos dialectos. Por ej.,

21 Es irrelevante que la $\alpha$ del aor. sea originaria o no (m vocalizada en gr. común, según la gramática tradicional, E. SCHWYZER, op. cit, I, 750; P. ChANTRAINE, Morphologie..., 176).

22 En cambio, H. BERMANN («Greek - $\sigma \alpha 1$ : Hittite -asha-» ZVS, 91, 1977, 231-239) da una explicación del inf. - $\sigma \alpha$ antitética a la nuestra: lo relaciona con el sufijo hetita de nombre verbal -asha- y propone reconstruir ${ }^{*}-\mathrm{sH}_{2}$-ei, en donde la laringal sería el reflejo de la $-\boldsymbol{h}$ - del mencionado sufijo $y^{*}$-ei la desinencia de dativo. Los inf. gr. en - $\alpha$ l serían en parte debidos al influjo analogico de $-\sigma \alpha \mathbf{~}$. 


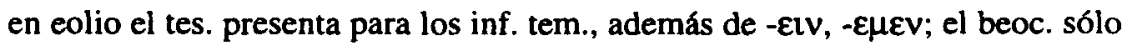
$-\varepsilon \mu \varepsilon v$, evidentemente tomada de los atem. El origen puede ser éste: la desaparición de la silbante en el inf. *-es-en dejaba en contacto las dos vocales * $\varepsilon \varepsilon v$ (tes. $-\varepsilon \imath v$, lesb. $-\eta v$ ); para evitar el hiato, $-\varepsilon v$ fue posteriormente sustituida por $-\mu \varepsilon v$ desinencia atemática, resultando $-\varepsilon \mu \varepsilon v$, que en beoc. desplazó definitivamente a la primera forma.

Como hemos visto (3., 6., 7.), en los inf. atem. -Evor se utilizaba cuando

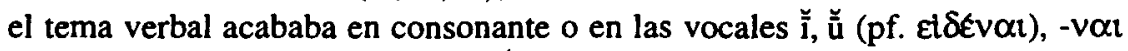
cuando terminaba en vocal (pres. i $\sigma \tau \alpha v \alpha \mathrm{l}$ ); por eso resultan a primera vista sorprendentes los inf. contractos de este tipo atestiguados en jón.-át. y arc. El punto de partida debió de ser la caída, en época postmicénica, de la wau inter-

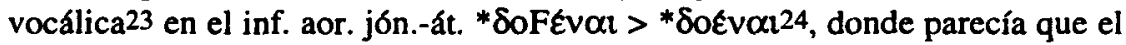
inf. - $\varepsilon v \propto a$ se unía directamente al tema $\delta 0$-. Sobre este modelo se crearon * $\vartheta \varepsilon$ $\varepsilon \vee \propto a$ y *' $\varepsilon-\varepsilon v \propto l$. La innovación se extendió luego al arc. 25 Una vez contraídas

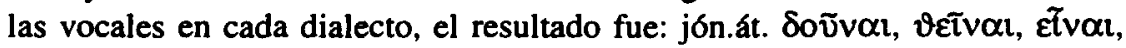

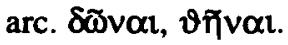

23 En jón.-át. sólo excepcionalmente hay testimonios de wau intervocálica. El arc. más conservador, presenta algunos, sobre todo en compuestos y formas verbales con aumento o reduplicación por influencia de las palabras simples o formas sin aumento o reduplicación, donde wau per-

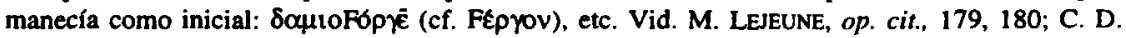
BUCK, The greek dialects, Chicago 19684, 48, 49; L. DuBOIs, Recherches sur le dialecte arcadien, Louvain-La-Neuve 1988, 53-57, 175, 176.

24 Ambas formas están, sin embargo, documentadas en chipriota. L. DuBoIs, op. cit., 175.

25 Para comprender mejor los hechos que aquí se exponen, será útil consultar la moderna visión de la dialectología griega (pero aplicable también a la de cualquier otra lengua) que A. LÓPEZ EIRE ofrece en su artículo «Las invasiones griegas y la Dialectologian, Homenaje a M. Vigil Pascual, Acta Salamanticensia, Salamanca 1989, 17-27. Entresacamos, por su interés, los siguientes párrafos (pp. 26, 27):

«... en las diversas áreas en que se hablaba griego jamás se hablo el "griego puro", "el griego arquetípico" que nunca existio, sino modalidades diferentes de griego distanciadas entre sí por sus específicas innovaciones pero unidas mutuamente por la conservación de rasgos linguísticos de épocas anteriores (los arcaísmos) y, a la vez, por su común participación en idénticas innovaciones. De esta manera, a los dialectos griegos no los trajeron a Grecia ya hechos, sino que se fueron gestando en Grecia; y no se hicieron de una vez, en aislamiento, y luego quedaron definitivamente configurados como tales, sino que se fueron configurando y conformando lentamente a base de un contacto continuo e ininterrumpido con otros dialectos. Así es como el jónico-ático comparte innovaciones con el lesbio y con el dorio y con el arcadio... Para explicar la génesis de los dialectos griegos, las invasiones ayudan poco y si, en cambio, estorban mucho. La dialectología nos informa de una larga, secular convivencia de los hablantes de griego los unos con los otrosw. 
9. De acuerdo con esta exposición, vamos a ensayar una cronología relativa así como una distinción de sustratos o niveles de lengua en el inf. gr. Estrato I: 1) *-en, 2) *-men. Estrato II: 3) *-es-en, 4) *-(e)s-dhai. Estrato III: 5) *-en-ai, 6) *-men-ai, 7) *-nai, 8) *-men(ai) tras consonante, 9) *-sai.

Los inf. de los estratos I y II pertenecen al período en que el grupo antepasado de los griegos se encontraba en contacto con la familia ide., mientras que los del III no suponen ya contacto alguno. A la lengua de este último nivel, que no es afectada ya por influencias ide. de ningún género, es a la que con mayor propiedad deberíamos denominar griego común.

En fin, hemos podido ver cómo, a partir de la reorganización de un núcleo primitivo, que resultó ambigua e inestable (3., 4.), dos tendencias contrapuestas han provocado la evolución del inf. gr.: por un proceso de diferenciación la lengua ha logrado distinguir entre inf. tem. y atem. (5.1., 5.3., 6.); gracias a la analogía el gr. ha conseguido caracterizar todo el bloque del inf. atem. frente al tem. (6.) y ella es asimismo la responsable de la creación de los inf. $-(\varepsilon) \sigma \vartheta \alpha \mathrm{l},(5.2$.$) y -\sigma \alpha \mathfrak{l}(7$.$) .$

Véase también J. MORALEJo, «Problemas actuales de la dialectología griega», Estudios medotológicos sobre la lengua griega, Universidad de Extremadura, Cáceres 1983, 53-68; F. R. ADRADOS, «La dialectología griega», Actualización científica en Filología Griega, Universidad Complutense, Madrid 1984, 219-237. 